\title{
Fecal Elastase: A Useful Test for Pancreatic Insufficiency?
}

\author{
Jo Tod $\cdot$ David Fine
}

Published online: 14 September 2010

(C) Springer Science+Business Media, LLC 2010

Pancreatic fecal elastase-1 (FE-1) has become the first-line test of pancreatic function. Our experience as a referral center for pancreatic disease suggests that in the minds of most gastroenterologists, a low FE-1 is diagnostic of pancreatic insufficiency. The paper by Evans and colleagues in the current issue of this journal highlights some weaknesses of that assumption in patients with Celiac disease.

FE-1, first described by Balo and Banga in 1950 [1], is one of a family of protease enzymes secreted by acinar cells. FE-1 is well documented in quantitative studies to be stable during intestinal transit and studies demonstrate significant correlation between fecal elastase and levels of other pancreatic enzymes such as duodenal lipase, amylase, trypsin, and bicarbonate concentration [2-6]. Fecal elastase is concentrated in human feces compared with pancreatic juice and is a simple, non-invasive and inexpensive test $[2,7,8]$. As a result, FE-1 has replaced "tube tests" and fecal fat measurement in most centers and has become the sole arbiter of pancreatic insufficiency for many. While low FE-1 is undoubtedly a marker for genuine pancreatic insufficiency, there is a longstanding debate about its specificity in the presence of other disorders.

Pancreatic disease is diagnosed on a combination of clinical history, abnormal pancreatic function tests, and radiological criteria [8]. Although subtle changes in exocrine function can be identified in patients with early pancreatic disease, overt steatorrhea does not occur until approximately $90 \%$ of the glandular function has been lost [9]. Direct function tests such as the secretin-cholecystokinin have the highest sensitivity and specificity and are the

J. Tod · D. Fine $(\bowtie)$

Southampton General Hospital, Tremona Road, Southampton, Hampshire SO16 1YD, UK

e-mail: davidrfine@gmail.com 'gold standard' [8]. They are, however, expensive, invasive, and time-consuming. FE-1 has been well characterized as a highly sensitive biomarker of moderate to severe pancreatic insufficiency with sensitivities of 73-100\% and specificities of $80-100 \%$ [3, 5, 10, 11]. Fecal elastase has also been shown to be a useful screening tool for children with primary pancreatic disorders, especially in cystic fibrosis, and in this setting has been well validated [3]. It is widely accepted however that FE-1 is not helpful in the diagnosis of mild to moderate exocrine pancreatic insufficiency with a low sensitivity $0-65 \%$ [4, 5, 7]. In addition, in both adults and children, FE-1 has been shown to be non-specific in differentiating non-pancreatic malabsorption from pancreatic malabsorption [12].

Two key limitations have been identified by the current literature on the clinical utility of fecal elastase-1:

1. Difficulties in diagnosing mild-moderate pancreatic insufficiency

2. Differentiating pancreatic from non-pancreatic malabsorption

The paper by Evans et al. highlights the second of these clinical challenges. In this paper, the first longitudinal study to follow-up adult celiac patients with low fecal elastase levels [13], 20 patients with diagnosed celiac disease who remained symptomatic with chronic diarrhea and a low FE-1 despite a gluten-free diet (GFD), were commenced on pancreatic enzyme therapy (PET). Patients were reassessed at 6 and 12 months, and then annual intervals over a period of 4 years. At each assessment, symptoms, weight, bowel habits, and FE-1 were recorded. No significant association was found between persistently low FE-1, age, sex, length of time between diagnosis of celiac disease and commencement of pancreatic enzyme supplementation, or adherence to GFD. Interestingly, FE-1 
levels were shown to increase over the study period, from a median of $90 \mu \mathrm{g} / \mathrm{g}$ stool (15-196) at diagnosis, to $212 \mu \mathrm{g} / \mathrm{g}$ stool $(15-402)$ at 6 months, and $365 \mu \mathrm{g} / \mathrm{g}$ stool (154$>500$ ) at more than 12 months. About 18 of the 20 participants had a significant long-term improvement in their chronic diarrhea with a reduction in median stool frequency from four per day to one per day $(p=<0.0001)$ and a reported subjective improvement in consistency and urgency. Eight patients stopped pancreatic enzyme replacement due to resolution of symptoms. Thirteen out of 20 participants consented to repeat duodenal biopsy and histology was compared to previous duodenal biopsies. Four patients had normal histology, two patients had increased intraepithelial lymphocytes with crypt hyperplasia, and seven patients still had evidence of villous atrophy. Eleven of 13 patients had histological improvement when compared to their index biopsy, and 2/11 were unchanged.

These results raise as many questions as they answer. As the authors discuss, dilution of the enzyme by excessive water in the stool is one possibility, i.e., an artifact of the test. If this were the case, one would not expect a symptomatic response to PET. A more intriguing possibility is that celiac disease disrupts the entero-pancreatic hormonal axis: the evidence for this is incomplete. Schappi et al. [14] compared FE-1 measurements to duodenal villous: crypt ratio and lamina propria inflammation in children with primary intestinal diseases including autoimmune enteropathy, post-infectious enteropathy, celiac enteropathy, short-gut syndrome, and Crohn's disease. Inflammation was found to correlate with FE-1 concentrations. In patients with low FE-1 results, $91.2 \%$ of 34 had evidence of mucosal inflammation within the duodenum. They concluded that functional pancreatic insufficiency is most likely to be due to reduction in pancreatic stimulation with impaired secretion of secretin or cholecystokinin by the inflamed mucosa. Several studies in children have supported the finding of low FE-1 levels in small intestinal disease including giardiasis, short-bowel syndrome, and celiac disease $[3,11]$.

Carroccio et al. however, used the secretin-caerulein test to assess pancreatic insufficiency in children with celiac disease before and after a prolonged gluten-free period [15]. They reported that prior to treatment, $22 \%$ had a reduction in secretion of one or more pancreatic enzymes, which was independent of the degree of intestinal mucosal damage. After treatment and normalization of the intestinal mucosa, no patient had pancreatic insufficiency. The impairment of response to direct hormonal stimulation in a proportion of these patients argues against a simple lack of enteric hormone release as the sole cause but suggests (at least in some cases) a longer-term effect of celiac disease, which might be mediated hormonally or via malnutrition but which is correctable by restoration of mucosal integrity. Secondary pancreatic insufficiency also is well recognized in conditions causing rapid intestinal transit such as in short-bowel syndrome or post-gastrectomy diarrhea.

Giovanni et al. reported low FE-1 levels in patients with inflammatory bowel disease, compared to controls. Low FE-1 correlated with disease activity, alterations in bowel habits (frequency of loose stools), but not with weight loss. FE-1 levels increased as IBD activity decreased, an observation that has been supported by other studies $[10,16]$.

Evans' study found that celiac patients with low FE-1 levels treated with pancreatic enzyme supplementation gained symptomatic improvement. Patients continued to receive treatment even after normalization of FE-1 levels. Eight patients stopped pancreatic enzyme supplementation as their symptoms resolved, suggesting that if true exocrine pancreatic insufficiency existed in this group, it was reversible and more likely to reflect a secondary pancreatic insufficiency. It is important, however, to consider the possibility of concomitant pancreatic insufficiency due to primary pancreatic pathology in this patient group. A large epidemiological trial in Sweden examining the risk of pancreatitis in 14,239 patients with celiac disease compared to age- and sex-matched individuals found an increased risk of all types of pancreatitis in patients with celiac disease $(p<0.01)$ [17]. Adjusting for socio-economic class, diabetes, alcohol-related disorders, and gallstone disease had no effect on risk estimates. This implies that patients with celiac disease may have an increased risk of pancreatic damage.

The limitations of the Evans study include the likelihood of confounding factors such as poor compliance with gluten-free diet and ongoing positive tissue transglutaminase (TTG) results. Equally, as FE-1 is not affected by pancreatic enzyme supplementation, compliance with treatment could not be verified. It would have been strengthened by the addition of a placebo control group, and by stopping the enzyme supplementation once FE-1 levels normalized. Nonetheless, it has confirmed that low FE-1 measurements are a common finding in patients with celiac disease and that they may be transient and therefore benign. In a small number of patients it has shown that FE-1 levels increased with an improvement in duodenal morphology and this is supported by other studies, suggesting the likelihood of secondary pancreatic insufficiency or possibly a dilutional effect. Pancreatic pathology may coexist in a minority of celiac patients and the mechanisms for this need to be further elucidated.

The most important message from this paper, however, is that FE-1 is limited in its ability to distinguish primary exocrine pancreatic insufficiency from other causes of diarrhea. Abnormal FE-1 levels need to be interpreted with 
caution, particularly in patients with pre-existing gastrointestinal disease. FE-1 can only be a part of the diagnostic algorithm and needs to be correlated with history, duodenal histology, and pancreatic imaging. It further highlights the need for new tests of pancreatic and intestinal function. This is a field that has been relatively neglected for 30 years and the time has come for the physiology and pathophysiology of digestion and absorption to be re-visited using current methodology.

\section{Key Points}

- Fecal elastase is valuable as a reproducible marker for severe exocrine pancreatic insufficiency.

- Fecal elastase never attains the sensitivity and specificity of the direct function tests.

- In the presence of intestinal inflammation or enteropathy, FE-1 must be interpreted with caution.

- An abnormal fecal elastase alone does not make the diagnosis of true pancreatic insufficiency in the absence of supporting imaging or direct function tests.

\section{References}

1. Balo J, Banga J. The elastolytic activity of pancreatic extracts. Biochem J. 1950;46:384-387.

2. Daftary A, Acton J, Heubi J, et al. Fecal elastase-1: utility in pancreatic function in cystic fibrosis. J Cyst Fibros. 2006;5: 71-76.

3. Beharry S, Ellis L, Corey M, et al. How useful is fecal pancreatic elastase 1 as a marker of exocrine pancreatic disease? J Pediatr. 2002;141:84-90.

4. Stevens T, Conwell D, Zuccaro G, et al. Analysis of pancreatic elastase-1 concentrations in duodenal aspirates from healthy subjects and patients with chronic pancreatitis. Dig Dis Sci. 2004; 49:1405-1411.

5. Loser C, Mollgaard A, Folsch U. Fecal elastase 1: A novel, highly sensitive, specific tubeless pancreatic function test. Gut. 1996; 39:580-586.

6. Stein J, Jung M, Sziegoleit A, et al. Immunoreactive elastase 1: clinical evaluation of a new noninvasive test of pancreatic function. Clin Chem. 1996;42:222-226.

7. Naruse S, Ishiguro H, Ko S, et al. Fecal pancreatic elastase: a reproducible marker for severe exocrine pancreatic insufficiency. J Gastroenterol. 2006;41:901-908.

8. Sonwalker S, Holbrook I, Phillips I, et al. A prospective, comparative study in the para-aminobenzoic acid test and fecal elastase 1 in the assessment of exocrine pancreatic function. Aliment Pharmacol Ther. 2003;17:467-471.

9. DiMagno E, Go V, Summerskill W. Relations between pancreatic enzyme outputs and malabsorption in severe pancreatic insufficiency. N Engl J Med. 1973;288:813.

10. Gullo L, Ventrucci M, Tomassetti P, et al. Fecal elastase 1 determination in chronic pancreatitis. Dig Dis Sci. 1999;44: 210-213.

11. Carroccio A, Verghi F, Santini B, et al. Diagnostic accuracy of fecal elastase 1 assay in patients with pancreatic maldigestion or intestinal malabsorption. Dig Dis Sci. 2001;46:1335-1342.

12. DiMagno E. A perspective on the use of tubeless pancreatic function tests in diagnosis. Gut. 1998;43:2-3.

13. Evans KE, Leeds JS, Morley S, Sanders DS. Pancreatic insufficiency in adult celiac disease: do patients require long-term enzyme supplementation? Dig Dis Sci. 2010. doi:10.1007/s10620-0101261-y.

14. Schappi M, Smith V, Cubitt D, et al. Fecal elastase 1 concentration is a marker of duodenal enteropathy. Arch Dis Child. 2002;86:50-53.

15. Carroccio A, Iacono G, Montalto G, et al. Exocrine pancreatic function in children with coeliac disease before and after a gluten-free diet. Gut. 1991;32:796-799.

16. Giovanni M, Dominici R, Molteni M, et al. Prevalence of pancreatic insufficiency inflammatory bowel disease. Assessment by fecal elastase-1. Dig Dis Sci. 2008;53:262-270.

17. Ludvigsson J, Montgomery S, Ekbom A. Risk of pancreatitis in 14,000 individuals with celiac disease. Clin Gastroenterol Hepatol. 2007;5:1347-1353. 